\title{
Duchesnea Phenolic Fraction Inhibits Tumor Growth through Restoring the Th1/Th2 Balance in U14 Cervical Cancer Bearing Mice
}

\author{
Bo Peng ${ }^{1,2}$, Qin Hu ${ }^{1,3}$, Lihua Sun ${ }^{1,4}$, Ying Chen ${ }^{1,2}$, Xinmin Liu ${ }^{*}$, Jianrong Li $^{2}$, Qi Chang ${ }^{1}$, \\ Liwei Wang ${ }^{1}$, Jintian Tang ${ }^{5}$ \\ ${ }^{1}$ Institute of Medicinal Plant Development, Chinese Academy of Medical Sciences, \\ Peking Union Medical College, Beijing, China \\ ${ }^{2}$ Institute of Chinese Materia Medica, China Academy of Chinese Medical Sciences, Beijing, China \\ ${ }^{3}$ The Laboratory of Virology and Pharmacology, College of Life Science and Bioengineering, \\ Beijing University of Technology, Beijing, China \\ ${ }^{4}$ Medical Experimental Center, China Academy of Chinese Medical Sciences, Beijing, China \\ ${ }^{5}$ Institute of Medicinal Physics \& Engineering, Tsinghua University, Beijing, China \\ Email: *liuxinminuae@yahoo.com.cn
}

Received September 26, 2011; revised November 6, 2011; accepted November 21, 2011

\begin{abstract}
Duchesnea indica (Andr.) Focke has been traditionally used to treat cancer in Asian countries for centuries. In the present study, transplanted U14 cervical cancer mouse model was used to evaluate the antitumor and immunomodulatory activity of Duchesnea phenolic fraction (DPF). ELISA and RIA assay were employed to measured the serum concentration of Th1/Th2 cytokines (IL-2, IL-4, IFN- $\gamma$ and TNF- $\alpha$ ). Administration with 0.25 g/kg, $0.5 \mathrm{~g} / \mathrm{kg}$ and $1 \mathrm{~g} / \mathrm{kg}$ DPF significantly reduced the tumor weight by $34.37 \%, 43.89 \%$ and $56.28 \%$, respectively, as compared to the tumor control group. Furthermore, the serum level of IL-2, IFN- $\gamma$ and TNF- $\alpha$ increased and IL-4 level decreased in a dose-dependent manner during DPF treatment, indicating that the antitumor activity of DPF may be associated with the decrease of TNF- $\alpha$ level and restoration of the balance of Th1/Th2 cell responses. These data suggested that DPF, a mixture of plant polyphenols, had potent anticancer activity which was in part accomplished by its immunomodulatory ability.
\end{abstract}

Keywords: Duchesnea indica (Andr.) Focke; Cervical Cancer; Th1/Th2 Cell Responses

\section{Introduction}

The immune system plays a critical role in surveillance and prevention of malignancy [1]. Based on the cytokine production, the immune response is classified into a cellular or humoral mediated response. The production of IL-2, IFN- $\gamma$ and TNF- $\alpha$ evokes Th1-type cellular response, while production of IL-4, IL-5, IL-6 and IL-10 stimulates Th2-type humoral immunity [2]. Recent studies have reported that the shift of Th1/Th2 balance toward Th2 dominance was a crucial factor in the malignancy stage, and restoration of the proper balance between Th1 to Th2 cells was perceived to be essential in the treatment of tumors [3]. Studies to date have demonstrated that immune response is the target for many therapeutic natural phytochemicals, including phenolics [4], flavonoids [4] and polysaccharide [5].

Duchesnea indica (Andr.) Focke and D. chrysantha (Zoll.

${ }^{*}$ Corresponding author. et Mor.) Miq, with the common name Indian-mock strawberry (IMH), has been documented as an anti-inflammatory, astringent and anticancer folk medicine. It is often used for cancer therapy alone or as a main ingredient in the formulas with traditional reputed benefits for the treatment of cancer in China and Japan. Previous studies have shown the anticancer activity of Duchesnea against many different types of cancer in both in vitro and in vivo settings [6-8]. Our recent work demonstrated that the phenolic extract of $D$. indica triggered mitochondria-dependent apoptosis and induced $\mathrm{S}$ phase arrest in cancer cells [7,9]. It also up-regulated lymphocyte proliferation stimulated by concanavalin A (Con A) and lipopolysaccharide (LPS) in U14 tumor mice [10]. However, scarce data are available regarding the immunomodulatory efficacy and mechanisms of Duchesnea in tumor bearing hosts.

Therefore, the present study focused on the anticancer activity of Duchesnea phenolic fraction (DPF) and its regulatory effect on the balance of Th1/Th2 cell responses in mice bearing U14 cervical tumors. 


\section{Materials and Methods}

\subsection{Plant Material and Preparation of DPF}

D. indica (Andr.) Focke, cultivated in Anhui province of China, was supplied by the company of Chinese Materia Medica in Beijing. The species was identified by Professor Bengang Zhang from the Institute of Medicinal Plant Development, Chinese Academy of Medical Sciences. A voucher specimen (number 20050443) is available in the herbarium of the institute.

The phenolic fraction of this plant, namely Duchesnea phenolic fraction (DPF), was prepared as described previously [7]. The total phenolic content of DPF was $48.03 \pm$ $0.07 \mathrm{~g}$ of catechin equivalents/100 g DPF using Folin-Ciocalteu assay.

\subsection{Cell Line and Culture}

The mouse cervical carcinoma U14 cell line was obtained from the Cell Bank of Institute of Basic Medical Sciences (Peking Union Medical College, Beijing, China). Cells were cultured in Dulbecco's modified Eagle's medium (DMEM; high glucose, Gibco) supplemented with 10\% fetal bovine serum (FBS, Hyclone).

\subsection{Animal Studies}

Female CD-1 mice (20 - 24 g) were obtained from Beijing Vital Laboratory Animal Technology (Beijing, China) and maintained in pathogen-free conditions, with food and water ad libitum. All procedures involving animals and their care were approved by the Animal Ethics Committee of Peking Union Medical College (Beijing, China) and conducted in compliance with "Guide of the Care and Use of Laboratory Animals” (NIH publication No. 86 - 23, revised 1996).

Animals were divided into six groups ( $\mathrm{n}=15$ per group). Mice were inoculated with $2 \times 10^{6} \mathrm{U} 14$ cervical cancer cells subcutaneously into the right flank. Sham mice were injected with saline vehicle. On the following day, mice were gavaged with sterile water (sham group and tumor control group) or DPF at doses of $0.25 \mathrm{~g} / \mathrm{kg}, 0.5 \mathrm{~g} / \mathrm{kg}$, and $1 \mathrm{~g} / \mathrm{kg}$ for 15 days. Cisplatin $\left(5 \times 10^{-4} \mathrm{~g} / \mathrm{kg}\right)$ was administered intraperitoneally as positive control every seventh day. After 15 days of treatment, mice were humanely sacrificed and tumors were collected and weighed. Tumor tissue was fixed for $\mathrm{H} \& \mathrm{E}$ staining and serum was obtained by centrifugation for $15 \mathrm{~min}$ at $2500 \mathrm{~g}$.

\subsection{Assessment of Serum Cytokine Levels}

The serum IL-4 and IFN- $\gamma$ levels were measured by the ELISA assay kit (R\&B company, USA), and TNF- $\alpha$, IL-2 and IL-10 levels were detected by the RIA assay kit (Beijing East Asian Institute of Immunology, China) according to the manufacturer's manuals.

\subsection{Statistical Analysis}

Data were expressed as mean \pm SEM. All statistical analyses were performed using GraphPad Prism 4.0 Software. Statistical significant differences between control and DPF-treated samples were determined by One-way ANOVA followed by Dunnett's test for post-hoc comparisons. In each case, P-value $<0.05$ was considered statistically significant.

\section{Results}

\subsection{Antitumor Effects of DPF on the Subcutaneous Tumor Model}

In vivo efficacy of DPF against cervical carcinoma was detected in the U14 tumor transplanted mouse model. On day 3 after tumor implantation, more than $80 \%$ of the mice had palpable flank tumors and no mice died during the course of the study. Oral administration of DPF at $0.25 \mathrm{~g} / \mathrm{kg}, 0.5 \mathrm{~g} / \mathrm{kg}$ and $1 \mathrm{~g} / \mathrm{kg}$ doses for 15 days significantly reduced the tumor weight by $34.37 \%, 43.89 \%$ and $56.28 \%$, respectively $(1.16 \pm 0.16 \mathrm{~g}$ in tumor control group versus $0.76 \pm 0.18 \mathrm{~g}$ in $0.25 \mathrm{~g} / \mathrm{kg}$ group, $0.65 \pm$ $0.15 \mathrm{~g}$ in $0.5 \mathrm{~g} / \mathrm{kg}$ group and $0.51 \pm 0.11 \mathrm{~g}$ in $1 \mathrm{~g} / \mathrm{kg}$ group, Figure 1).

H \& E staining was performed to monitor the morphological changes of tumors. After DPF treatment, tumors showed significantly apoptotic changes, including cell shrinkage, nuclear condensation and fragmentation, deeply eosinophilic cytoplasm surrounded by a clear "halo", and formation of apoptotic bodies (Figure 2).

\subsection{Effect of DPF on Serum Cytokine Levels in U14 Tumor Mice}

To evaluate the effect of DPF on the balance of Th1/Th2

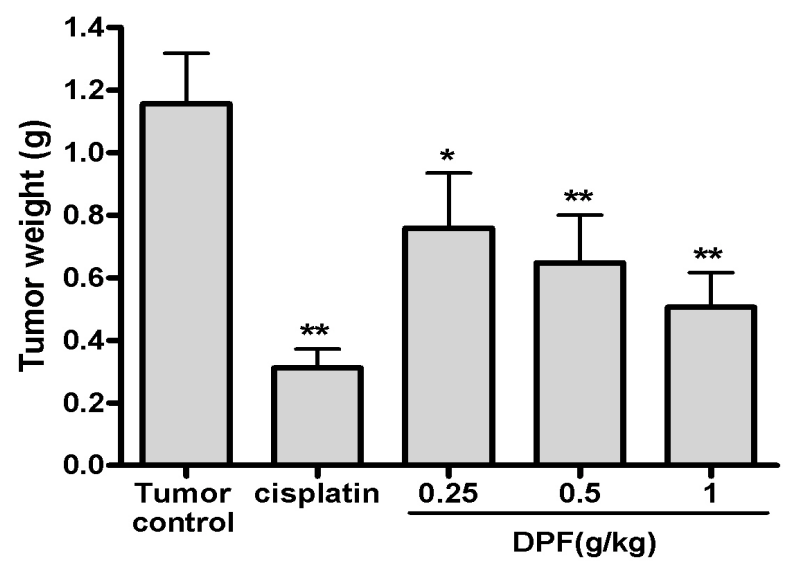

Figure 1. Antitumor effect of DPF in mice bearing cervical U14 tumors. Mice were implanted sc with $2 \times 10^{6}$ U14 cells. On the following day, mice were treated with water (Tumor control), cisplatin or 0.25 - $1 \mathrm{~g} / \mathrm{kg}$ doses of DPF. After treatment for 15 days, tumors were harvested and weighed. 


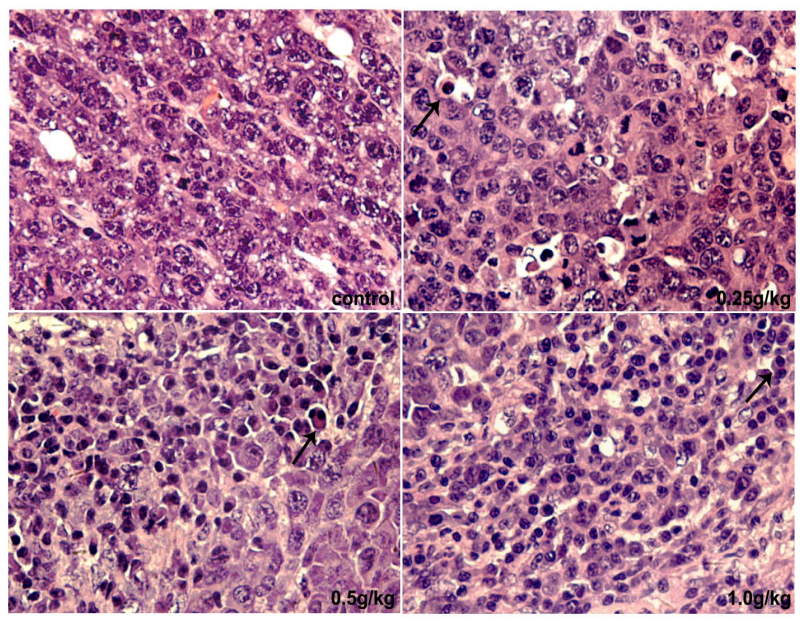

Figure 2. H \& E staining of tumor. 0.25 - $1 \mathrm{~g} / \mathrm{kg}$ doses of DPF induced apoptosis in U14 tumors (arrowheads indicate apoptotic cells; magnification, $\times 400)$.

cell responses, the levels of serum Th1 cytokines (IL-2 and IFN- $\gamma$ ) and serum Th2 cytokines (IL-4 and IL-10) were measured by ELISA or RIA assay (Table 1). As shown in Table 1, IL-2 and IFN- $\gamma$ levels were significantly decreased and IL-4 level was markedly increased in U14 tumor mice ( $\mathrm{P}<0.05$ vs. sham group). These results indicated that the Th cell responses tended toward Th2 dominance in U14 mice. After treatment with DPF for 15 days, IL-2 and IFN- $\gamma$ levels were significantly increased and IL-4 was back to 21 - $26 \mathrm{pg} / \mathrm{ml}$, suggesting that Th2 dominance is readily transformed to Th1 polarization.

Serum TNF- $\alpha$ level in U14 mice was significantly upregulated compared to the sham group, and dose-dependently down-regulated in the presence of DPF $(\mathrm{P}<0.05)$.

\section{Discussion}

In this study, DPF at doses of 0.25 - $1 \mathrm{~g} / \mathrm{kg}$ significantly inhibited tumor growth in a dose-dependent manner (Figure 1) with no obvious side effects (data not shown) in mice. $\mathrm{H}$ \& E staining analysis has revealed the apoptosis induced by DPF in U14 tumors, which is consistent with our previous results showing that the mechanisms of its antitumor activity in vivo and in vitro were associated with S phase arrest and induction of mitochondria-de- pendent apoptosis [7,9]. In addition, we detected that DPF could up-regulate splenocytes proliferation stimulated by $\mathrm{T}$ and B mitogens (Con A and LPS) in U14 tumor mice [10]. Thus, the aim of our present study was to further analyze the effect of DPF on the serum cytokines, which play a critical role in stimulating the immune defense system against tumor cells.

TNF- $\alpha$ is a multifunctional cytokine involved in immunity, cancer and inflammation via two receptors. It has conflicting roles in cancer, as a tumor necrotic factor or a promoting growth factor [11]. TNF- $\alpha$ has the ability to induce apoptosis and exerts antitumor effects in both preclinical and clinical settings [12]. On the other side, TNF- $\alpha$ provides a survival signal for cancer cells, deregulated TNF- $\alpha$ expression within the tumor microenvironment appears to favor malignant cell invasion, migration and metastasis formation [13]. Our data showed that DPF significantly decreased the TNF- $\alpha$ level of the U14 tumor mice in a dose-dependent manner (Table 1). It indicated that the antitumor activity of DPF might be associated with the down-regulation of TNF- $\alpha$.

It is widely considered that the breakage of Th1/Th2 cell responses is a crucial factor in the malignancy stage. Recent work has demonstrated that the Th1/Th2 balance shifts toward Th2 dominance in the cervical cancer bearing state [3]. To explore the immunomodulatory mechanism of protection in DPF-treated mice, we analyzed the cytokines production of Th1 and Th2 immune cells, which are essential to anti-tumor immunity, including IL-2, IFN- $\gamma$, IL-4 and IL-10. In this study, Th1 cytokine (IL-2 and IFN- $\gamma$ ) was markedly down-regulated, while Th2 cytokine (IL-4) was significantly up-regulated in U14 tumor bearing mice compared to sham mice. These data supported the idea that the Th1/Th2 balance shifted toward Th2 dominance in cervical cancer hosts. As shown in Table 1, DPF significantly increased the Th1 cytokine levels and reduced the Th2 cytokine levels, indicating that the predominance of Th2 cytokines was transformed to Th1 polarization in the presence of DPF and DPF has the capacity to restore the balance of Th1/Th2 cell responses. IL-10 is a Th2 cytokine and has conflicting roles in cancer. Previous study reported that increased expression of IL-10 is responsible for the progression and invasion of cervical

Table 1. Effect of DPF on the serum TNF- $\alpha$, IFN- $\gamma$, IL-2, IL-4 and IL-10 in U14 tumor mice.

\begin{tabular}{ccccccc}
\hline \multirow{2}{*}{ Group } & \multirow{2}{*}{ Dose $(\mathrm{g} / \mathrm{kg})$} & \multicolumn{5}{c}{ Serum cytokines concentration } \\
\cline { 3 - 7 } & & TNF- $\alpha(\mathrm{pg} / \mathrm{ml})$ & IFN- $\gamma(\mathrm{pg} / \mathrm{ml})$ & $\mathrm{IL}-2(\mathrm{ng} / \mathrm{ml})$ & $\mathrm{IL}-4(\mathrm{pg} / \mathrm{ml})$ & $\mathrm{IL}-10(\mathrm{pg} / \mathrm{ml})$ \\
\hline Sham mice & - & $0.69 \pm 0.08$ & $37.75 \pm 0.43$ & $0.99 \pm 0.16$ & $20.63 \pm 1.75$ & $34.97 \pm 1.45$ \\
Tumor mice & - & $0.97 \pm 0.05^{\#}$ & $29.11 \pm 3.27^{\#}$ & $0.60 \pm 0.17^{\#}$ & $43.30 \pm 5.17^{\# \#}$ & $33.74 \pm 2.19$ \\
Cisplatin & $5 \times 10^{-4}$ & $0.98 \pm 0.14$ & $37.33 \pm 1.22$ & $0.72 \pm 0.09$ & $14.30 \pm 1.76^{* * \#}$ & $34.40 \pm 1.55$ \\
& 0.25 & $0.72 \pm 0.08$ & $36.64 \pm 1.23$ & $1.43 \pm 0.15^{*}$ & $25.77 \pm 5.63^{* *}$ & $29.96 \pm 1.08$ \\
DPF treatment & 0.5 & $0.64 \pm 0.11^{*}$ & $38.82 \pm 1.83^{*}$ & $1.70 \pm 0.33^{* *}$ & $21.25 \pm 4.96^{* *}$ & $30.31 \pm 1.48$ \\
& 1 & $0.40 \pm 0.07^{* *}$ & $45.19 \pm 5.95^{* *}$ & $1.66 \pm 0.25^{* *}$ & $21.19 \pm 2.60^{* *}$ & $28.30 \pm 1.27$ \\
\hline
\end{tabular}

Data were shown as mean \pm SEM of fifteen mice in each group. ${ }^{*} \mathrm{P}<0.05 ;{ }^{* *} \mathrm{P}<0.01$ versus tumor mice; ${ }^{*} \mathrm{P}<0.05$; ${ }^{\# \#} \mathrm{P}<0.01$ versus sham mice. 
carcinoma [14], while the preclinical evidence demonstrated that IL-10 was associated with tumor regression [15]. In the present study, there was no change in serum IL-10 level by implantation of U14 cancer cells or DPF administration (Table 1). Further work is required to determine the effect of DPF on IL-10 level.

In conclusion, our data suggested that DPF, a mixture of plant polyphenols, had potent anticancer activity and its action was associated with the immunomodulatory effects, including activating cellular and humoral immune responses and restoring the balance between Th1 and Th2 cell responses. Further investigation is warranted to specify its immunomodulatory components and evaluate their anticancer actions.

\section{Acknowledgements}

This work was supported by a European Commission project grant (230232), a National specialized project grant (2009ZX09103-335) and a ICMM project grant (Z02070).

\section{REFERENCES}

[1] R. W. Melvold and R. P. Sticca, "Basic and Tumor Immunology: A Review," Surgical Oncology Clinics of North America, Vol. 16, No. 4, 2007, pp. 711-735. doi:10.1016/j.soc.2007.08.003

[2] S. Romagnani, "Th1/Th2 Cells," Inflammatory Bowel Diseases, Vol. 5, No. 4, 1999, pp. 285-294. doi:10.1002/ibd.3780050410

[3] A. Sharma, M. Rajappa, A. Saxena and M. Sharma, “Cytokine Profile in Indian Women with Cervical Intraepithelial Neoplasia and Cancer Cervix," International Journal of Gynecological Cancer, Vol. 17, No. 4, 2007, pp. 879-885. doi:10.1111/j.1525-1438.2007.00883.x

[4] N. Nair, S. Mahajan, R. Chawda, C. Kandaswami, T. C. Shanahan and S. A. Schwartz, "Grape Seed Extract Activates Th1 Cells in Vitro," Clinical and Diagnostic Laboratory Immunology, Vol. 9, No. 2, 2002, pp. 470-476.

[5] V. E. Ooi and F. Liu, "Immunomodulation and AntiCancer Activity of Polysaccharide-Protein Complexes,” Current Medicinal Chemistry, Vol. 7, No. 7, 2000, pp. 715-729.

[6] J. Y. Duan, X. P. Liu and Q. Li, "Research on the Effect of Duchesnea indica (Andr.) Focke,” Pharmacology and Clinics of Chinese Materia Medica, Vol. 14, 1998, p. 28.

[7] B. Peng, Q. Chang, L. W. Wang, Q. Hu, Y. Q. Wang, J. T. Tang and X. M. Liu, "Suppression of Human Ovarian SKOV-3 Cancer Cell Growth by Duchesnea Phenolic Fraction Is Associated with Cell Cycle Arrest and Apoptosis," Gynecologic Oncology, Vol. 180, No. 1, 2008, pp. 173-181. doi:10.1016/j.ygyno.2007.09.016

[8] M. Shoemaker, B. Hamilton, S. H. Dairkee, I. Cohen and M. J. Campbell, "In Vitro Anticancer Activity of Twelve Chinese Medicinal Herbs,” Phytotherapy Research, Vol. 19, No. 7, 2005, pp. 649-651. doi:10.1002/ptr.1702

[9] B. Peng, Q. Hu, X. M. Liu, L. Wang, Q. Chang, J. Li, J. Tang, N. Wang and Y. Wang, "Duchesnea Phenolic Fraction Inhibits in Vitro and in Vivo Growth of Cervical Cancer through Induction of Apoptosis and Cell Cycle Arrest," Experimental Biology and Medicine (Maywood), Vol. 234, No. 1, 2009, pp. 74-83. doi:10.3181/0806-RM-204

[10] B. Peng, Q. Hu, L. W. Wang, Y. Q. Wang, Q. Chang, J. T. Tang, X. M. Liu, Y. Chen and H. X. Dang, "Study on Anticancer Function of Phenolic Extract from Duchesnea Indica and Its Immunological Mechanism,” Chinese Pharmacological Bulletin, Vol. 23, No. 8, 2007, pp. 1007-1010.

[11] R. M. Locksley, N. Killeen and M. J. Lenardo, “The TNF and TNF Receptor Superfamilies: Integrating Mammalian Biology,” Cell, Vol. 104, No. 4, 2001, pp. 487-501. doi:10.1016/S0092-8674(01)00237-9

[12] R. Horssen, T. L. M. Hagen and A. M. M. Eggermont, "TNF- $\alpha$ in Cancer Treatment: Molecular Insights, Antitumor Effects, and Clinical Utility,” Oncologist, Vol. 11, No. 4, 2006, pp. 397-408. doi:10.1634/theoncologist.11-4-397

[13] S. Mocellin and D. Nitti, "TNF and Cancer: The Two Sides of the Coin," Frontiers in Bioscience, Vol. 13, 2008. pp. 2774-2783. doi:10.2741/2884

[14] R. K. Bhairavabhotla, V. Verma, H. Tongaonkar, S. Shastri, K. Dinshaw and S. Chiplunkar, "Role of IL-10 in Immune Suppression in Cervical Cancer,” Indian Journal of Biochemistry \& Biophysics, Vol. 44, No. 5, 2007, pp. 350-356.

[15] S. Mocellin, F. M. Marincola and H. A. Young, "Interleukin-10 and the Immune Response against Cancer: A Counterpoint," Journal of Leukocyte Biology, Vol. 78, No. 5, 2005, pp. 1043-1051. doi:10.1189/jlb.0705358 